\title{
On the mechanism of void growth and the effect of straining mode in ductile materials
}

\author{
Guo-Chen Li*, Xian-Wu Ling, Huang Shen \\ Laboratory for Nonlinear Mechanics of Continuous Media, Institute of Mechanics, \\ Academia Sinica, 15 Zhong Guan Cun Road, 100080, Beijing, PR China
}

Received in final revised form 10 June 1999

\begin{abstract}
Finite element analysis is employed to investigate void growth embedded in elastic-plastic matrix material. Axisymmetric and plane stress conditions are considered. The simulation of void growth in a unit cell model is carried out over a wide range of triaxial tensile stressing or large plastic straining for various strain hardening materials to study the mechanism of void growth in ductile materials. Triaxial tension and large plastic strain encircling around the void are found to be of most importance for driving void growth. The straining mode of incremental loading which favors the necessary strain concentration around void for its growth can be characterized by the vanishing condition of a parameter called "the third invariant of generalized strain rate". Under this condition, it accentuates the internal strain concentration and the strain energy stored/dissipated within the material layer surrounding the void. Experimental results are cited to justify the effect of this loading parameter. (C) 2000 Elsevier Science Ltd. All rights reserved.
\end{abstract}

Keywords: A. Voids and inclusions; B. Elastic-plastic material; C. Finite elements

\section{Introduction}

The analytic formulations given by Rice and Tracey (1969) and Gurson (1977) have been widely used to estimate the ductile damage caused by void growth in materials. These models do help understand the deterioration of the mechanical behavior of materials being voided.

\footnotetext{
* Corresponding author.

E-mail address: ligchen@cc5.imech.ac.cn (Guo-Chen Li).
} 
However, years afterwards, discrepancies have been found between the predictions of these models and experimental results or those based on concise finite element modeling. The reasons can be attributed to (a) the influence of strain softening or secondary void damage in the matrix material around primary void ( $\mathrm{Li}$ and Howard, 1983; Li et al., 1989; Brocks et al., 1995); (b) plastic flow localization due to non-uniform void distribution (Ohno and Hutchinson, 1984; Becker, 1987; Magnusen et al., 1988; Becker and Smelser, 1994); (c) the effect of void shape on void growth and ductility (Li, 1985a; Becker et al., 1989; Yee and Mear, 1996); (d) voiding instability in elastic-plastic solids (Koplik and Needleman, 1988; Huang et al., 1991); (e) the three dimensional effects (Hom and McMeeking, 1989; Worswick and Pick, 1990) and others such as, the effects of void cluster size (Benson, 1995) and the matrix compressibility on void growth (Briottet et al., 1996).

Tvergaard (1981) and Needleman and Tvergaard (1984) tried to modify Gurson's model by adding more parameters so as to bring shear band bifurcation predictions of the Gurson constitutive relation into closer agreement with corresponding results of full numerical analysis and to take into account the effects of rapid void coalescence at failure. A sophisticated process was implemented to determine the parameters for making comparisons of the calculations between the modified Gurson model and those of experimental results (Becker et al., 1988). As another approach, $\mathrm{Li}$ (1985b) proposed to use dilatational constitutive equations to account for the voiding damage. Computer simulations of axisymmetric bars and microstructural measurements were employed to determine the two tangent moduli included in the dilatational constitutive equations. The whole process was also complicated for comparing theoretical modelings with the experimental results of notched bars, cracked three-point bend specimens (Li et al., 1992) and the case of shear band bifurcation (Li and Zhu, 1995).

This general background stimulates our interest to focus attention again on void growth analysis, since more accurate modeling and simpler implementation are expected to be based on deeper understanding of its mechanism. A more direct reason comes from the question raised by the experiments done by Zhang et al. (1990), in which they tested the void growth in dual-phase steel sheets subjected to three straining paths. They measured the growth of the sizes of void in the sheets and found that it was the plane strain loading condition that yielded the fastest void growth, although the triaxial tension and plastic strain loaded on the sheet were both lower than those in the case of biaxial tension. So far, this phenomenon is not able to be explained by the existing models. Hence we may ask, besides the plastic equivalent strain (or equivalently saying the equivalent stress) and the triaxial tension, should there be a third loading factor that could be affecting the growth of void?

The main goal of this paper is focused on the effect of this third parameter, which is initially suggested in the following as "the third invariant of generalized strain rate." The vanishing condition of this parameter is numerically proved to have quite similar effect as that of large triaxial stresses to yield concentrated plastic strains encircling around the void. This mechanism is of vital necessity for moving the boundary layer of void. Two dimensional modeling (before the most preferable three dimensional one that should be followed afterwards) is carried out in this 
paper to make primary studies of the mechanism of void growth in a wide range of loading for various strain hardening materials.

\section{Finite element modeling}

\subsection{Cell model under proportional straining}

Matrix material containing periodically distributed voids can be modeled by considering a unit cell embedding one void. A quadrant of this cell model is shown in Fig. 1(a) for analyzing axisymmetric (r, z) and plane stress (x, y) cases. The radius of the initial void is given as $R_{0}$, when the half lengths along the main directions of the unit cell are initially equal and normalized as a unit. The shape of void is spherical in the case of using axisymmetric cell, but is circular throughout the thickness when the plane stress model is employed.

Proportional straining is enforced on the cell whose boundaries are free from shear stress and kept straight to ensure continuity; so as to take into account the interaction between voids distributed periodically. During an incremental displacement loading, we have

$$
\frac{\mathrm{d} U_{\mathrm{r}}}{L_{\mathrm{r}}}=\alpha \frac{\mathrm{d} W_{\mathrm{z}}}{L_{\mathrm{z}}} \quad\left(\text { i.e. } \mathrm{d} \bar{\varepsilon}_{\mathrm{r}}=\alpha \mathrm{d} \bar{\varepsilon}_{\mathrm{z}}\right)
$$

for the axisymmetric model, in which $L_{\mathrm{r}}$ and $U_{\mathrm{r}}$ are, respectively, the current radius and the radial displacement along the radial direction $r$ of the cell, both being normalized by the initial cell radius. Correspondingly, $L_{\mathrm{z}}$ and $W_{\mathrm{z}}$ are assigned to those parameters of the axial direction $z . \alpha$ is a proportional straining parameter, controlling the overall straining ratio between $\bar{\varepsilon}_{\mathrm{r}}$ and $\bar{\varepsilon}_{\mathrm{z}}$ of the cell. Similarly, we take

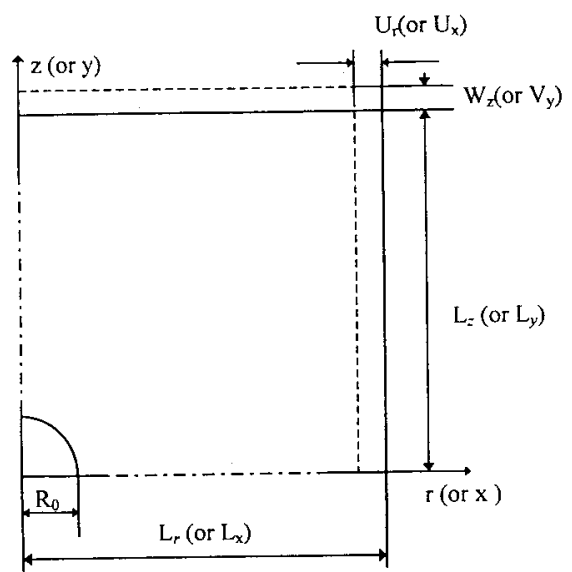

(a)

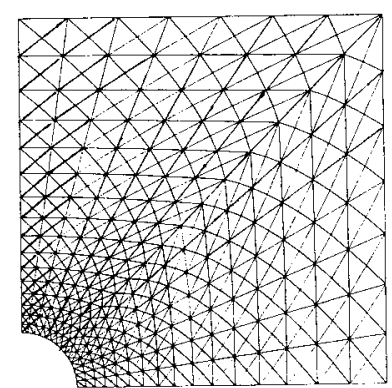

(b)

Fig. 1. A quadrant of cell model and its finite element mesh. 


$$
\left.\frac{\mathrm{d} U_{\mathrm{x}}}{L_{\mathrm{x}}}=\alpha \frac{\mathrm{d} V_{\mathrm{y}}}{L_{\mathrm{y}}} \quad \text { (i.e. } \mathrm{d} \bar{\varepsilon}_{\mathrm{x}}=\alpha \mathrm{d} \bar{\varepsilon}_{\mathrm{y}}\right)
$$

for plane stress model associated with similar sense in the nominations of the parameters $L_{\mathrm{x}}, U_{\mathrm{x}}, L_{\mathrm{y}}$ and $V_{\mathrm{y}}$. Hence, Eq. (2) represents a proportional straining between overall strains $\bar{\varepsilon}_{\mathrm{x}}$ and $\bar{\varepsilon}_{\mathrm{y}}$.

\subsection{Constitutive equations}

The elastic-plastic material that encircles the void is taken to follow a generalized form of the Prandtl-Reuss equations in the case of large strain analysis, that is, the relationship between the Jaumann rate $\mathcal{D} / \mathcal{D} t$ of Kirchhoff stress $\tau_{\mathrm{ij}}$ and the deformation rate $D_{\mathrm{kl}}$ [which is related to the velocity $V_{\mathrm{k}}$ as $D_{\mathrm{kl}}=\frac{1}{2}\left(V_{\mathrm{k}, 1}+V_{1, \mathrm{k}}\right)$ ]

$$
\frac{\mathcal{D}}{\mathcal{D} t} \tau_{\mathrm{ij}}=L_{\mathrm{ijk} \mathrm{l}} D_{\mathrm{kl}}
$$

with the stiffness tensor

$$
L_{\mathrm{ijk} l}=\frac{E}{1+v}\left[\frac{1}{2}\left(\delta_{\mathrm{ik}} \delta_{\mathrm{j} 1}+\delta_{\mathrm{il}} \delta_{\mathrm{jk}}\right)+\frac{v}{1-2 v} \delta_{\mathrm{ij}} \delta_{\mathrm{kl}}-\frac{3}{2 \sigma_{\mathrm{e}}^{2}} \frac{E}{E_{\mathrm{te}}} \frac{S_{\mathrm{ij}} S_{\mathrm{kl}}}{\frac{2}{3}(1+v)+\frac{E}{E_{\mathrm{te}}}}\right]
$$

In Eq. (3b), $E$ and $v$ are, respectively, Young's modulus and Poisson's ratio; $\sigma_{\mathrm{e}}=$ $\left(\frac{3}{2} S_{i j} S_{j i}\right)^{1 / 2}$ is equivalent stress with the deviatoric stress written as $S_{\mathrm{ij}}$, $\delta_{\mathrm{ij}}$ denotes Kronecker delta; $E_{\mathrm{te}}^{(\mathrm{p})}$ is the plastic tangent modulus along the equivalent stress-strain curve $\left(\sigma_{\mathrm{e}}-\varepsilon_{\mathrm{e}}^{(\mathrm{p})}\right)$. Assuming the matrix material to follow the power law and neglecting the difference between the total equivalent strain $\varepsilon_{\mathrm{e}}$ and its plastic part $\varepsilon_{\mathrm{e}}^{(\mathrm{p})}$, we take

$$
\frac{E_{\mathrm{te}}^{(\mathrm{p})}}{E}=n\left(\frac{\varepsilon_{\mathrm{e}}}{\varepsilon_{\mathrm{y}}}\right)^{n-1}
$$

for strain hardening material with various values of exponent parameter $n$ (given as $n=0.05,0.10$ and 0.20 ) and $\varepsilon_{\mathrm{y}}$ is the yield strain (taken as $\varepsilon_{\mathrm{y}}=0.002$ ).

\subsection{Functional formulation for solution}

The solution of the problem is obtained through using the up-dated Lagrangian formulation given by McMeeking and Rice (1975) for elastic-plastic, large strain analysis. It is to minimize the functional

$$
\prod=\frac{1}{2} \int_{v}\left[\frac{\mathcal{D} \tau_{\mathrm{ij}}}{\mathcal{D} t} D_{\mathrm{ij}}-\sigma_{\mathrm{ij}}\left(2 D_{\mathrm{ik}} D_{\mathrm{jk}}-V_{\mathrm{k}, \mathrm{i}} V_{\mathrm{k}, \mathrm{j}}\right)\right] \mathrm{d} v-\int_{S} \dot{T}_{\mathrm{i}} V_{\mathrm{i}} \mathrm{d} s
$$

where $\sigma_{\mathrm{ij}}$ is the true stress and $\dot{T}_{\mathrm{i}}$ denotes the rate of surface traction. In our computations, the elongation ( $W_{\mathrm{z}}$ or $V_{\mathrm{y}}$ ) along the major loading axis is chosen for the 
generalized time $t$. The procedure of computations within each incremental $\Delta t$ of time follows the explanation given by $\mathrm{Li}$ and Howard (1983). This non-iterative updated procedure, as has been checked, may induce an error in the prediction of the local stresses in the finite elements less than $0.8 \%$, when compared with the corresponding stresses that should be yielded by the local strains accumulated in those elements, in case the increments of loading are taken carefully and small enough in the computations. The prediction of such precision would be considered as acceptable for numerical analysis. The number of incremental steps needed to complete each calculation varies from 500 to 3000 , depending on the ductility of the sample. We mostly stopped the calculation of each sample when any local equivalent strain in the periphery zone of the void reached $\varepsilon_{\mathrm{e}}=1$, so as to have the same basis for comparing the local strain distributions of all the examples considered.

\subsection{Finite element meshes and justifications}

A quadrant of the cell model, as shown in Fig. 1(b), was subdivided into 224 quadrilaterals with four crossed triangular elements in each of it, altogether consisted of 896 constant strain triangular elements for employing the finite element method. Finer mesh of $1680(420 \times 4)$ elements was used to check the precision of the former calculations with regard to the maximum loading stresses and the current void volume fractions. In both aspects, the differences are limited within $1 \%$. The finer mesh was also used for the case when the instability of void growth was concerned.

As our main concern in this paper, the calculations of void growth based on direct integration of current void volume were also checked by an analytic formula, derived by Koplik and Needleman (1988) for axisymmetric cell model of matrix materials associated with plastic incompressibility. The current void volume fraction can be expressed as

$$
f_{\mathrm{v}}=1-\left(1-f_{\mathrm{v} 0}\right)\left[1+\frac{3(1-2 v)}{E} \bar{\sigma}_{\mathrm{m}}\right] \frac{V}{V_{0}}
$$

where $f_{v}$ and $f_{v 0}$ represent the current and the initial void volume fraction, respectively, $\bar{\sigma}_{\mathrm{m}}\left(=\frac{1}{3} \bar{\sigma}_{\mathrm{kk}}\right)$ is the overall mean stress loaded on the cell model, $V$ and $V_{0}$ are, respectively, the current and the initial volume of the cell. The two methods for calculating void volume fraction $f_{v}$ produce very close results within the ranges of our calculations.

\section{Void growth in axisymmetric cell model}

\subsection{Relationships in axisymmetric cell model under proportional straining}

During proportional straining at the boundaries of an axisymmetric cell model embedding a spherical void, the overall principal true strain $\bar{\varepsilon}_{\mathrm{r}}$ and $\bar{\varepsilon}_{\mathrm{Z}}$ can be calculated by the following relationships: 


$$
\begin{aligned}
& \bar{\varepsilon}_{\mathrm{z}}=\ln \left(1+W_{\mathrm{z}}\right) \\
& \bar{\epsilon}_{\mathrm{r}}=\ln \left(1+U_{\mathrm{r}}\right)=\alpha \bar{\epsilon}_{\mathrm{z}}
\end{aligned}
$$

then the overall equivalent strain $\bar{\epsilon}_{\mathrm{e}}$ is

$$
\bar{\varepsilon}_{\mathrm{e}}=\frac{2}{3}\left(\bar{\varepsilon}_{\mathrm{z}}-\bar{\varepsilon}_{\mathrm{r}}\right)
$$

The overall equivalent stress $\bar{\sigma}_{\mathrm{e}}$ and mean stress $\bar{\sigma}_{\mathrm{m}}$ can be related to the average axial stress $\bar{\sigma}_{\mathrm{z}}$ and the average radial stress $\bar{\sigma}_{\mathrm{r}}$ along the boundaries of the cell model as

$$
\begin{aligned}
& \bar{\sigma}_{\mathrm{e}}=\left|\bar{\sigma}_{\mathrm{z}}-\bar{\sigma}_{\mathrm{r}}\right| \\
& \bar{\sigma}_{\mathrm{m}}=\frac{\bar{\sigma}_{\mathrm{z}}+2 \bar{\sigma}_{\mathrm{r}}}{3}
\end{aligned}
$$

The bar over the symbols represents the sense of overall response of the cell so as to distinguish with the local stress or strain within the matrix.

\subsection{The effects of triaxiality and large plastic strain around void}

The initial void volume fraction is $f_{\mathrm{v} 0}=0.225 \%$, i.e. $R_{0}=0.15$. We calculated for $\alpha=1,0.5,0.3,0.0,-0.3,-0.45,-0.48,-0.50,-0.75$ and -1 . The case of $\alpha=1$, $\bar{\sigma}_{\mathrm{e}}=\bar{\varepsilon}_{\mathrm{e}}=0$, is a pure triaxial stressing condition. When $\alpha \leqslant-0.5$, the overall mean stress turns from tension to compression.

Fig. 2(a)-(c) show the growth of the void volume fraction $f_{v}$ with respect to the overall equivalent strain $\bar{\varepsilon}_{\mathrm{e}}$ for various strain-hardening exponents $n=0.05,0.1$ and 0.2 . Correspondingly, the relationships between the triaxial parameter $T\left(=\bar{\sigma}_{\mathrm{m}} / \bar{\sigma}_{\mathrm{e}}\right)$ and $\bar{\varepsilon}_{\mathrm{e}}$ are also given in Fig. 3(a)-(c). In view of these two groups of figures, it is obvious that void growth mainly depends on the triaxiality conditions. After the turning condition of $\alpha=-0.5$, once the sign of $T$ changes, then void stops growing or shrinks.

The contours of constant local equivalent strain $\varepsilon_{\mathrm{e}}$ are shown in Fig. 4 with the current shapes of the cell and void for $n=0.2$ and $a=1.0,-0.3,-0.45$ and -0.50 . The strain distributions along the radial axis and the central axis are also depicted along the borders of the quadrant with the largest point referring to $\varepsilon_{\mathrm{e}}=1$. Voids grow almost spherically with slight flattening along the radial direction $r$ within the range of $-0.3 \leqslant \alpha \leqslant 1$. The overall equivalent strain $\bar{\epsilon}_{\mathrm{e}}$ keeps to be zero in the case of $\alpha=1.0$, plastic area covers the void as a spherical layer leaving large zone of elasticity in the outer part of the matrix. Elastic unloading can happen in the matrix and is sooner or later accompanied by dropping of the overall stress from its maximum point in the range of $-0.45<\alpha<1$. Void shape changes to prolate type with slight amount of growth, when $-0.5<\alpha<-0.45$, then only small zone of elastic unloading can be seen above the crest of the void. Beyond this transition range no elastic unloading is seen if $-1.0<\alpha<-0.5$, and the void shrinks spherically. Correspondingly, no sharp drop of stresses are likely to occur within the range of $-1.0<\alpha<-0.45$.

To summarize, we can say that triaxiality is the driving force for enlarging void, since no void can grow without it. On the other hand, a layer of large plastic strain 

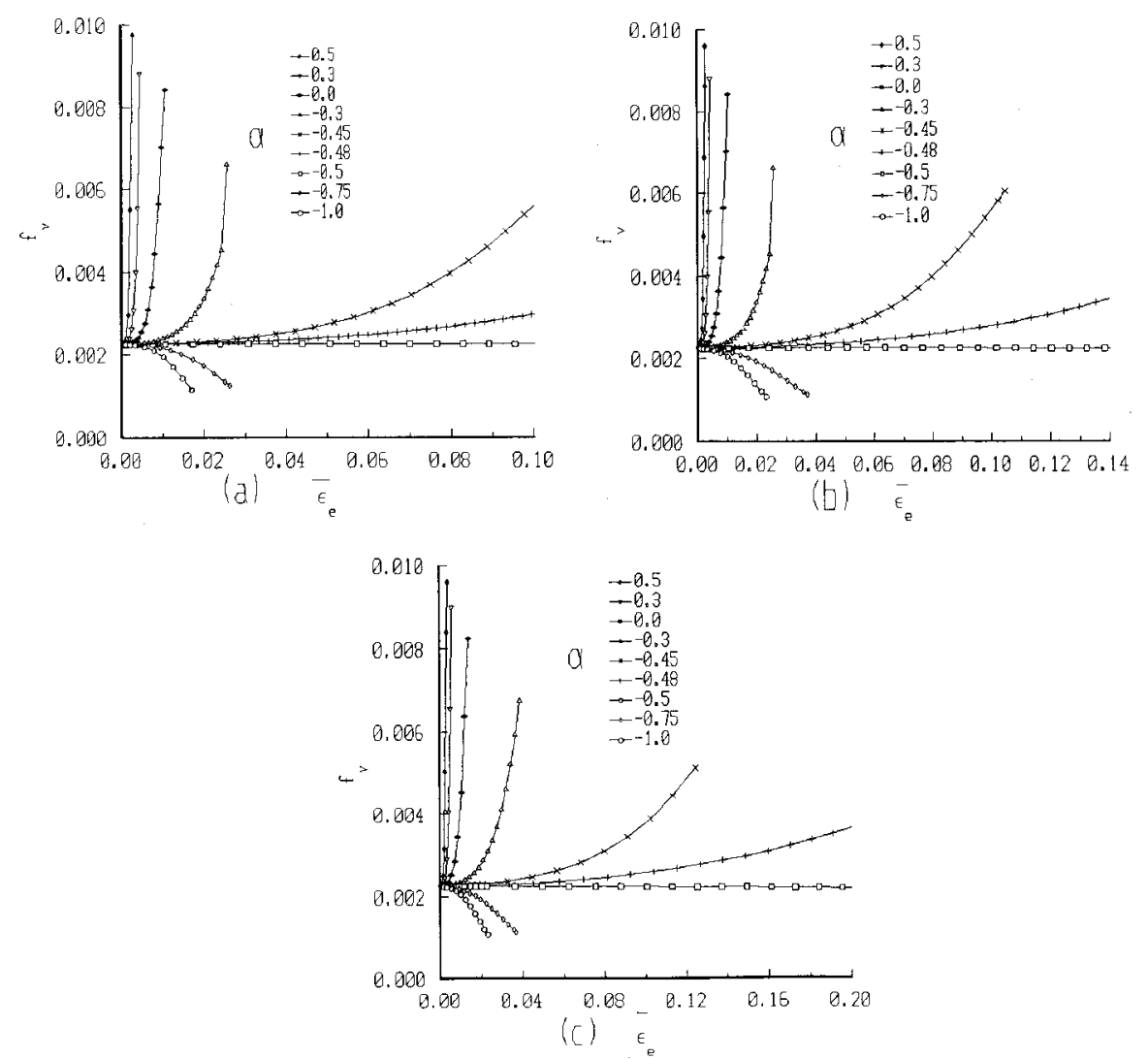

Fig. 2. The growth of void volume fraction $f_{\mathrm{v}}$ with respect to the overall equivalent strain $\bar{\varepsilon}_{\mathrm{e}}$ in the axisymmetric cells with various strain hardening exponents. (a) $n=0.05$, (b) $n=0.10$, (c) $n=0.20$.

around the void is a favorable and necessary environment for the void to grow. Therefore, compared with the situation of the cell which has harder matrix, softer material yields larger void fraction under the same amount of overall strain $\bar{\varepsilon}_{\mathrm{e}}$. Within the range of the samples studied above, none of these two factors can be omitted when void growth is concerned. The extreme case of $\alpha=1.0$ typically demonstrates this nature. While the case of $\alpha=-0.5$ yields no void growth as the triaxiality turns to nil, although the internal matrix is covered thoroughly by large plastic strains. Unless new voids are nucleated, no void volume fraction can be increased under the conditions of $\alpha \leqslant-0.5$.

\subsection{The effects of the evolution of void shape}

Figs. 3 and 4 also indicate that the shape of void is strongly dependent on the triaxiality condition $T$ of the cell model subjected to straining. The transition of the 

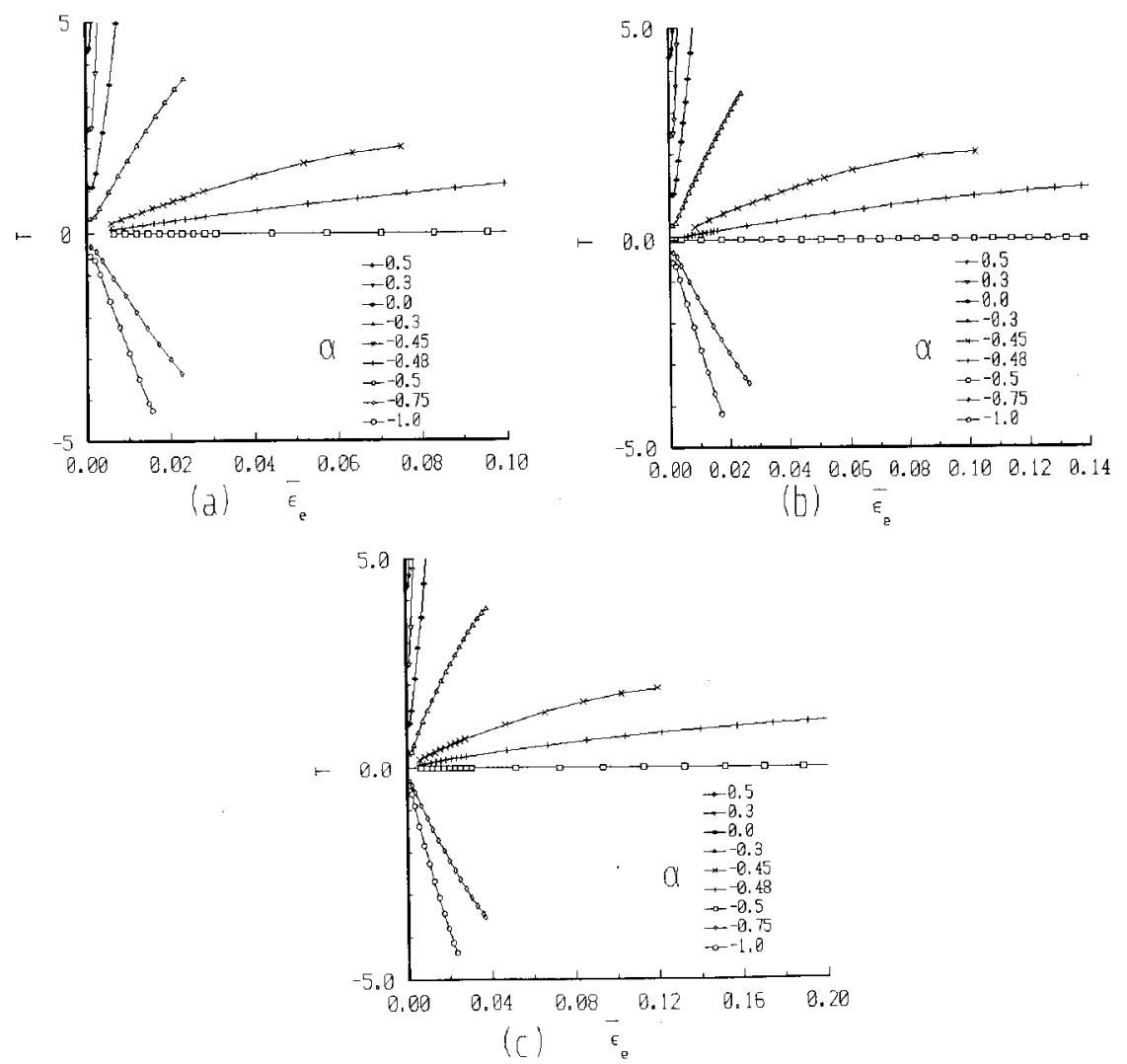

Fig. 3. The relationship between the triaxiality parameter $T$ and overall equivalent strain $\bar{\varepsilon}_{\mathrm{e}}$ of axisymmetric cells with various strain hardening exponents. (a) $n=0.05$, (b) $n=0.10$, (c) $n=0.20$.

shape from slight oblate to prolate occurs within the range of $-0.45<\alpha<-0.30$. This trend is similar to the results given by Koplik and Needleman (1988) and Brocks et al. (1995), although their loading process is to keep triaxiality $T$ constant.

The ductility of material is much gained from the transition. This could be attributed to that the prolate shape of void would be equivalent to a decrease of the effective size of a spherical void. This equivalency can be explained by the similarity in the damage effects caused by initially prolate or oblate voids with those of their corresponding void which has spherical shape with the same horizontal radius as theirs. Li (1985a) [published in Chinese but with a short English presentation in Li (1997)] and Becker et al. (1989) have come to the same conclusion from their independent studies that the failure of cell model should be related to the void radius along the horizontal direction, rather than to the void volume itself. Li (1985a) proposed the maximum loading point as the occurrence of failure, while Becker et al. (1989) used the instability of void growth for failure. 


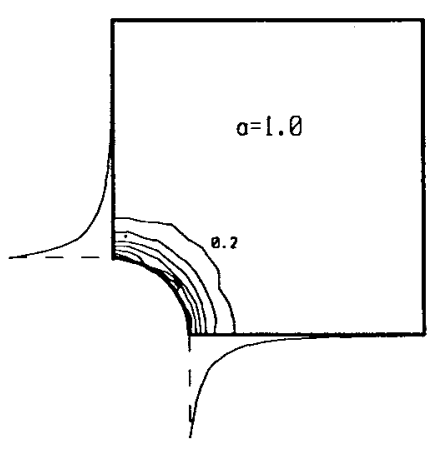

$\bar{\varepsilon}_{e}=0$.

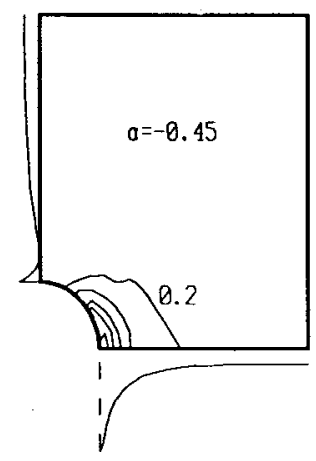

$\bar{\varepsilon}_{e}=0.140$

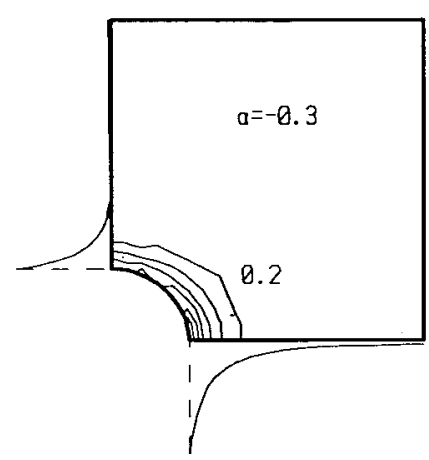

$\bar{\varepsilon}_{e}=0.043$

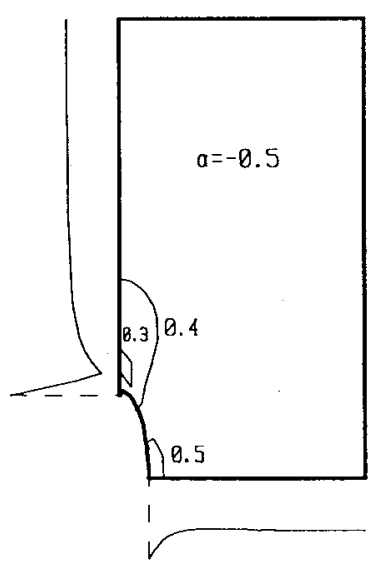

$\bar{\varepsilon}_{e}=0.440$

Fig. 4. The contours of constant local strain $\varepsilon_{\mathrm{e}}$ (with 0.1 grading) in axisymmetric cells $(n=0.20)$, when $\left(\varepsilon_{\mathrm{e}}\right)_{\max }=$ 1 at (a) $\bar{\varepsilon}_{\mathrm{e}}=0(\alpha=1)$, (b) $\bar{\varepsilon}_{e}=0.043(\alpha=-0.3)$, (c) $=0.140(\alpha=-0.45)$, and (d) $=0.440(\alpha=-0.5)$.

\subsection{The effects of straining mode}

To exemplify the important role played by the large plastic strain around void and to correlate this effect to the overall straining mode of the cell, we intend to study the constant $T$ modeling used by Koplik and Needleman (1988) and Brocks et al. (1995). It was shown by Brocks et al. (1995) that the calculation results of the cell model were strongly influenced by the finite element formulations under the lower triaxiality condition $(T=1)$. It seems to us that the uniqueness of solution is then questionable, we therefore only choose $T=2$ and 3 as samples for investigating the instability of void growth. It is necessary to unravel the mechanism which causes the void to grow rapidly, when the straining mode approaches to an uniaxial condition; since triaxiality $T$ is constant and the overall equivalent strain $\bar{\varepsilon}_{\mathrm{e}}$ varies very little.

As the material in the matrix of the cell model is expected to undergo much larger strain than those in the previous cases, we have to use finer mesh for the computations 
(i.e. 1680 constant strain triangular elements) to avoid deterioration that could be caused by those extremely distorted elements during numerical calculations.

In order to keep the loading triaxiality constant, we had to adjust the proportional straining parameter $\alpha$ of Eq. (1) with respect to the overall equivalent strain $\bar{\varepsilon}_{\mathrm{e}}$, in such a manner as shown in Fig. 5(a). When $\alpha$ is positive, it denotes that the lateral boundary of the cell is expanding. Conversely, it shrinks if $\alpha$ turns to negative. Both samples ( $T=2$ and 3 with $n=0.2$ ) have the same ending state of $\alpha$ approaching zero. The abrupt turning point is marked by a solid circle in Fig. 5(a) and (b), and is referred to as the occurrence of instability. Larger critical values of $f_{v \mathrm{c}}$ is needed in $T=2$ than that of $T=3$ to reach the instability point.

Actually, once approaching to this point, large zone of elastic unloading occurs in outer region of the cell within a very small extension of further elongation. This is in the same way as that predicted by Koplik and Needleman (1988). Subsequently, an exponential growth of void can be seen in Fig. 5(b) and the corresponding distributions of local equivalent strain at the instability points are given in Fig. 6. Large strain concentration occurs around the void and strain localizes in the ligament between neighboring voids with elastic unloading in the outer region of the matrix portion. This is a favorable environment for rapid growth of void as demonstrated previously and the turning of the parameter $\alpha$ to zero should be the stimulating factor that incurs the consequence.

A general formulation for this limiting case can be expressed in a generalized rate form under the condition of incremental loading. That is

$$
\dot{\bar{\varepsilon}}_{1} \dot{\bar{\varepsilon}}_{2} \dot{\bar{\varepsilon}}_{3}=0
$$

where 1, 2 and 3 are referring to the three main axes of strain rate in continuum, and the cell model in Fig. 1(a) is representing the detailed microstructure of this continuum.

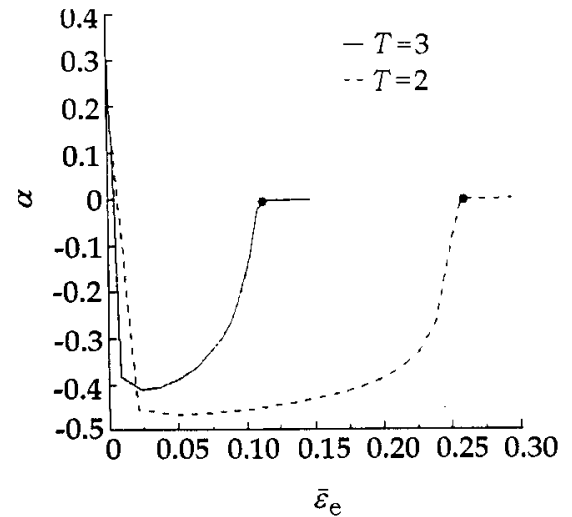

(a)

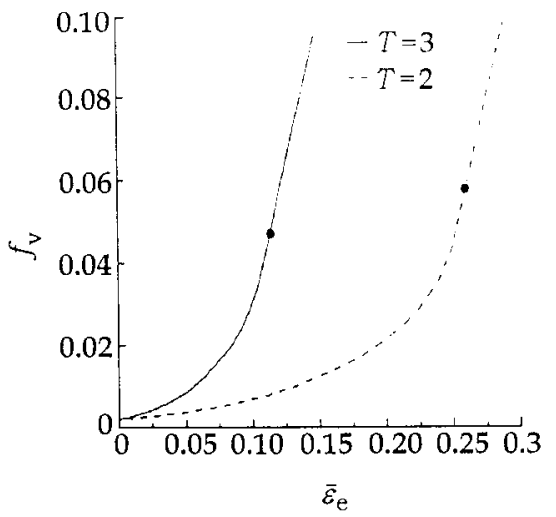

(b)

Fig. 5. (a) The variation of the proportional straining parameter $\alpha$ with respect to overall equivalent strain $\bar{\varepsilon}_{\mathrm{e}}(n=0.20)$ and (b) the growth of void volume fraction $f_{\mathrm{v}}$ with respect to overall equivalent strain $\bar{\varepsilon}_{\mathrm{e}}$ in axisymmetric cells loaded by constant triaxiality $T=2$ and $3(n=0.20)$. 
Eq. (8) implies that if any main strain rate keeps to be zero (except the meaningless case of having three of them equal to zero) it would be a dangerous condition of inducing rapid void growth. We will see in the next section that criterion (8) can also be applied to plane stress modeling of void growth. We may refer to this criterion as the vanishing condition of the third invariant of generalized strain rate (the rate is counted on the change with respect to the generalized time).

\section{Void growth in plane stress cell model}

\subsection{Considerations in plane stress cell model}

Although using a three dimensional cell with an initially spherical void would be the best for examining void growth under plane-stress loading, a two dimensional substitute can also be proved to be convincing for the explanation of the effect of straining mode observed in the tests of sheet metal forming before the occurrence of instability. That is to say, no localized thinning should occur in the plane stress cell model. This consideration is carefully taken into account so as to limit our computations not to run to too far an extent. Also limit our modeling as acceptable only to the surface layer of the metal sheet.

A quadrant of the plane stress cell model and its finite element discretizaton are the same as those of the axisymmetric cases and are shown in Fig. 1(a) and (b). The overall equivalent strain and mean strain can be written in integration forms as

$$
\bar{\varepsilon}_{\mathrm{e}}=\int_{0}^{t} \mathrm{~d} \bar{\varepsilon}_{\mathrm{e}}
$$

and
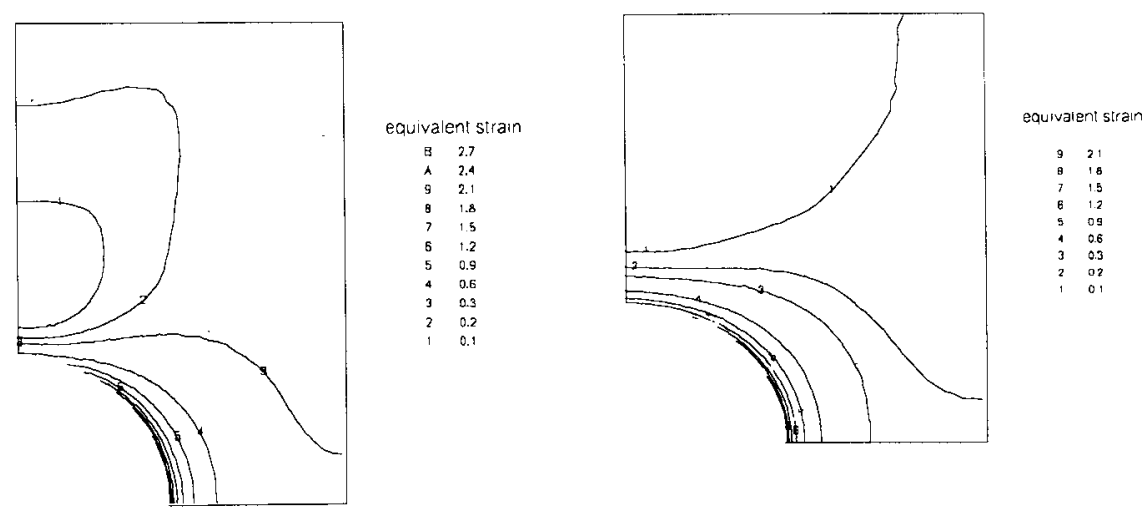

$$
\bar{\varepsilon}_{e}=0.260(\mathrm{~T}=2)
$$

$$
\bar{\varepsilon}_{e}=0.114(\mathrm{~T}=3)
$$

Fig. 6. The contours of constant local strain $\varepsilon_{\mathrm{e}}$ in the axisymmetric cells $(n=0.20)$ loaded by constant triaxiality $T=2\left(\bar{\varepsilon}_{\mathrm{e}}=0.260\right)$ and $T=3\left(\bar{\varepsilon}_{\mathrm{e}}=0.114\right)$ at the instability of void growth. 


$$
\mathrm{d} \bar{\varepsilon}_{\mathrm{e}}=\frac{\sqrt{2}}{3}\left[\left(\mathrm{~d} \bar{\varepsilon}_{\mathrm{x}}-\mathrm{d} \bar{\varepsilon}_{\mathrm{y}}\right)^{2}+\left(\mathrm{d} \bar{\varepsilon}_{\mathrm{y}}-\mathrm{d} \bar{\varepsilon}_{\mathrm{z}}\right)^{2}+\left(\mathrm{d} \bar{\varepsilon}_{\mathrm{z}}-\mathrm{d} \bar{\varepsilon}_{\mathrm{x}}\right)^{2}\right]^{1 / 2}
$$

In Eq. (9), $\mathrm{d} \bar{\varepsilon}_{\mathrm{x}}$ and $\mathrm{d} \bar{\varepsilon}_{\mathrm{y}}$ are the incremental loading strains given by Eq. (2), $\mathrm{d} \bar{\varepsilon}_{\mathrm{z}}$ is the incremental strain responded along the thickness and is counted as an average over the whole matrix area. The overall equivalent stress $\bar{\sigma}_{\mathrm{e}}$ and the mean stress $\bar{\sigma}_{\mathrm{m}}$ can be calculated by using

$$
\bar{\sigma}_{\mathrm{e}}=\left(\bar{\sigma}_{\mathrm{x}}^{2}-\bar{\sigma}_{\mathrm{x}} \bar{\sigma}_{\mathrm{y}}+\bar{\sigma}_{\mathrm{y}}^{2}\right)^{1 / 2} \quad \bar{\sigma}_{\mathrm{m}}=\frac{\bar{\sigma}_{\mathrm{x}}+\bar{\sigma}_{\mathrm{y}}}{3}
$$

where $\bar{\sigma}_{\mathrm{x}}$ and $\bar{\sigma}_{\mathrm{y}}$ are the average stresses along the boundary sections.

To check the validity of this two dimensional modeling, we calculated the pure shear condition. The boundaries are so controlled as to yield uniform tensile displacement along the $x$-direction but uniform compression on the $y$-direction. The two overall stresses along the boundaries are kept to have the same absolute values but opposite in sign as that done by Hom and McMeeking (1989) in their three dimensional void model. The initial void areal proportion is taken as $6.5 \%$, same as the initial void volume fraction used by Hom and McMeeking (1989). The overall true stress-strain curve is shown in the Appendix which is very near to the three dimensional finite-element predictions given by Hom and McMeeking (1989). The distribution of the local equivalent strain in the cell are quite similar to those given by Hom and McMeeking at the middle cross-section of their cell model before the overall axial straining is too large (i.e. $\dot{\varepsilon}_{\dot{a}} \leqslant 0.15$ ).

\subsection{Comparisons among various straining modes}

In Fig. 7(a) are shown the relationships between the void areal fraction $f_{\mathrm{a}}$ and the overall equivalent strain $\bar{\varepsilon}_{\mathrm{e}}$ for $\alpha=1.0,0$ and -0.45 with the strain hardening expo-
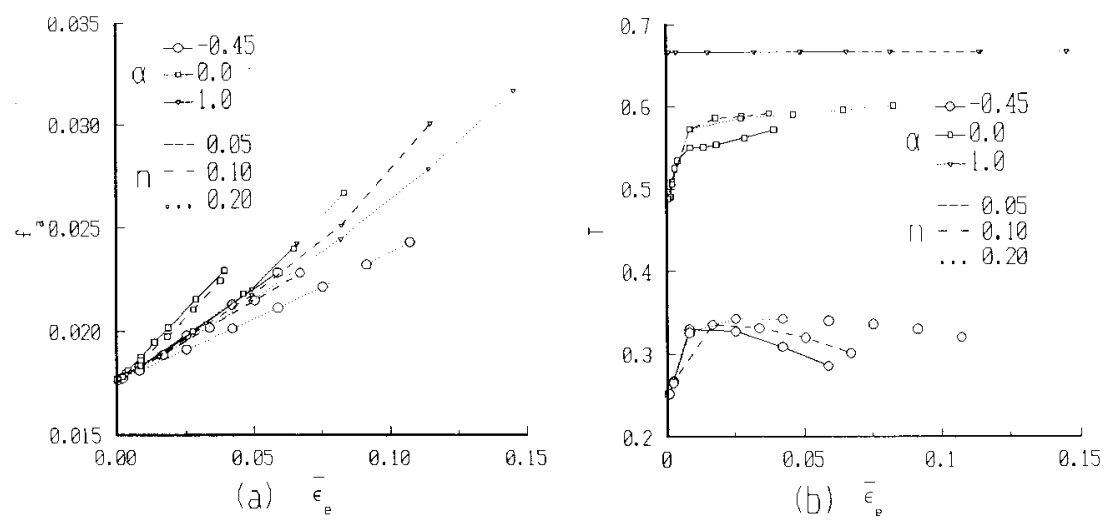

Fig. 7. (a) The growth of void areal fraction $f_{\mathrm{a}}$ with respect to overall equivalent strain $\bar{\varepsilon}_{\mathrm{e}}$ and (b) the relationship between the triaxiality parameter $T$ and overall equivalent strain $\bar{\varepsilon}_{\mathrm{e}}$ in the plane stress cells with various strain hardening exponents $n=0.05,0.10$ and 0.20 . 
nent $n=0.05,0.10$ and 0.20 . The corresponding conditions of the triaxiality $T=\bar{\sigma}_{\mathrm{m}} /$ $\bar{\sigma}_{\mathrm{e}}$ are given in Fig. 7(b). We can find that void growth is indeed fastest under the plane strain mode of loading $(\alpha=0)$, even faster than the biaxial case $(\alpha=1.0)$, although its triaxiality level is lower. This trend is in agreement with the experimental observation to be cited later and also provides justifications of using plane stress cell modeling to explain the phenomena in plane sheet testings.

Based on the computation results, we may think that besides the enlarging influence of triaxial tension on void, there must be some other mechanism which also favors its growth. The plane stress cell modeling proves again that plastic strain concentration around the void can also be a dominating factor. Let us define a strain concentration factor as

$$
\varepsilon_{\mathrm{s}}=\left(\varepsilon_{\mathrm{e}}\right)_{\text {maximum }} / \bar{\varepsilon}_{\mathrm{e}}
$$

which is the ratio between the maximum value of the local equivalent strain $\varepsilon_{\mathrm{e}}$ in the matrix and the overall equivalent strain $\bar{\varepsilon}_{\mathrm{e}}$ loaded on the cell. Table 1 gives a list of the values of the strain concentration factor $\varepsilon_{\mathrm{s}}$ at the same comparison basis of having the maximum local strain $\varepsilon_{\mathrm{e}}=1$; for $\alpha=1,0$ and -0.45 with $n=0.05,0.10$ and 0.20 . In all cases, the straining condition of $\alpha=0$ yields the largest value of $\varepsilon_{\mathrm{s}}$ and it becomes that the softer the matrix (smaller $n$ ) the larger the value of strain concentration factor. As can be seen from Fig. 7(b), the difference of triaxiality between the two cases of $\alpha=0$ and $\alpha=1$ is not large, then the influence of large plastic flow around the void can become overwhelming. Hence straining mode $(\alpha=0)$ comes into effect through its influence on the internal strain distribution around the void. It also proves the characterization of this mode by using criterion (8) is meaningful, and the use of an incremental (rate) form is more preferable for plastic analysis.

\subsection{The stimulating mechanism in the plane strain mode of straining}

Concentration of large plastic strain not only plays the role as an adjoint factor of voiding but also stimulates void growth, since larger equivalent strain lowers down the plastic stiffness around the void. The dependence of void growth on the plastic stiffness of material has already been demonstrated in the samples of both the axisymmetric models (Figs. 2 and 3) and the plane stress cases [Fig. 7(a) and (b)]. Softer matrix (with smaller value of $n$ ) can have larger void volume fraction $f_{\mathrm{v}}$ or areal fraction $f_{\mathrm{a}}$ than the harder one (with larger value of $n$ ) under the same overall equivalent strain $\bar{\varepsilon}_{e}$ with even smaller triaxiality $T$.

Table 1

Strain concentration factor $\varepsilon_{\mathrm{s}}$ when local equivalent strain $\varepsilon_{\mathrm{e}}=1$

\begin{tabular}{lrrr}
\hline$\alpha \backslash n$ & 0.05 & 0.1 & 0.2 \\
\hline-0.45 & 16.92 & 13.17 & 8.32 \\
0 & 23.87 & 19.46 & 11.26 \\
1 & 9.86 & 7.43 & 4.96 \\
\hline
\end{tabular}


We may further prove in the following examples that only an encircling layer around the void is mainly effective for counting the dependence of void growth on material stiffness. Fig. 8(a) demonstrates the comparison between matrix having single phase material (as boundary has the radius of $2 R_{0}$ as demonstrated in the corner of the figures and $n=0.1$ in the outer phase). The two materials yield almost the same condition of void growth [see Fig. 8(a)], although the two-phase model (with harder outer phase) may bring about a little higher triaxiality [see Fig. 8(b)]. If we use $n=0.1$ for the single phase model, then computation results show that its void growth will be lower than the two phase one used here, since the latter one has a softer inner phase. The two models have almost the same conditions for triaxiality as most part of the material in the two-phase model has the same strain hardening exponent $(n=0.1)$ as that of the single phase one. The use of a "fictitious" two-phase material is to prove that void growth is strongly dependent on the stiffness of the layer surrounding the void rather than on the block material apart from it. Then large plastic strains within this surrounding layer would play an equivalent role as softening the material to yield larger void growth. Although it is difficult to have "any existing experiment against which to test these predictions", (Fleck and Hutchinson, 1997), it is the same conclusion from both studies that void growth should have strong dependence on the hardness of a shell surrounding the void. Fleck and Hutchinson (1997) used "strain gradient" mechanism to harden the shell, while we apply a two-phase model to the voided cell to soften the layer. The approaches are different but results of implication are equally satisfactory.

The above results inspire us to observe more closely on the inner area of the matrix. By considering the ratio $\rho_{\text {se }}$ between the strain energy stored/dissipated in the inner area and that of the whole matrix, when the cell model is composed of the same material of
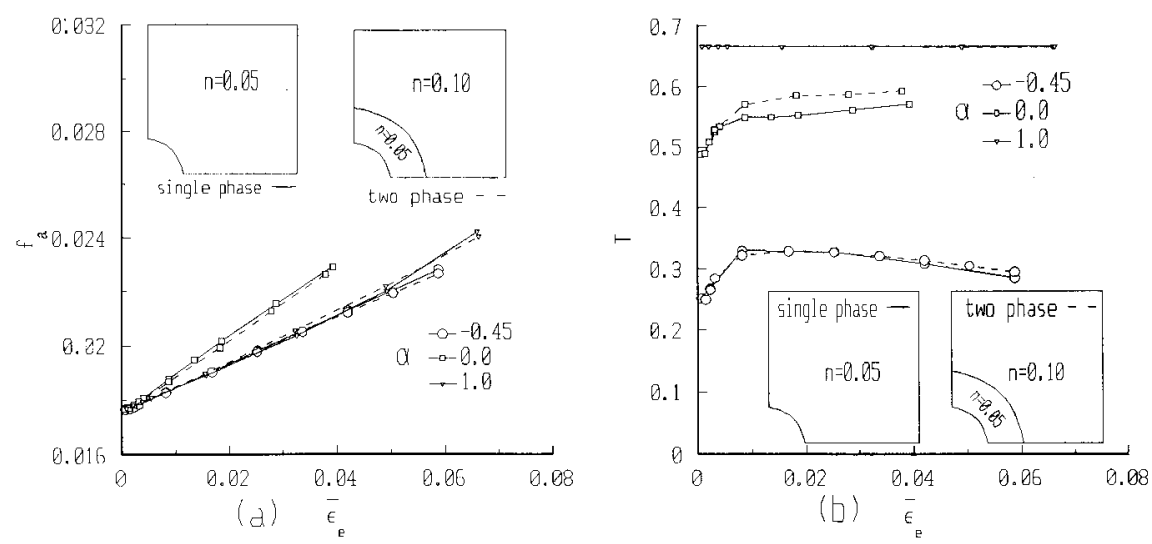

Fig. 8. Comparison between the matrix having single phase material $(n=0.05)$ and a two-phase material (inner phase $n=0.05$ with its exterior radius as $2 R_{0}$, outer phase $n=0.10$ ). (a) The growth of void areal fraction $f_{\mathrm{a}}$ with respect to overall equivalent strain $\bar{\varepsilon}_{\mathrm{e}}$. (b) The triaxiality $T$ versus the overall equivalent strain $\bar{\varepsilon}_{\mathrm{e}}$. 
$n=0.05,0.10$ and 0.20 . The inner area $\mathrm{A}$ has its exterior radius as $2 R_{0}$, leaving the remaining part of the matrix as $\mathrm{B}$. Hence, we have the strain energy ratio

$$
\rho_{\mathrm{se}}=\int_{0}^{t} \int_{A} \sigma_{\mathrm{ij}} D_{\mathrm{ij}} \mathrm{d} s \mathrm{~d} t / \int_{0}^{t} \int_{\mathrm{A}+\mathrm{B}} \sigma_{\mathrm{ij}} D_{\mathrm{ij}} \mathrm{d} s \mathrm{~d} t
$$

we counted the variation of $\rho_{\mathrm{se}}$ with respect to the void growth parameter $f_{\mathrm{a}} / f_{\mathrm{a} 0}$ which is the ratio between the current and initial areal fractions for proportional straining controlled as $\alpha=1,0$ and -0.45 . The results are depicted in Fig. 9, which indicates that the case of plane strain loading has the largest proportion of strain energy for its inner area and this situation would likely transfer more energy for void growth.

These examples demonstrate that the overall straining mode, which can be characterized by criterion (8), has its influence on not only the local strain concentration that affects the plastic stiffness around the void but also the distribution of strain energy within the matrix. Stronger strain concentration brings about lower plastic stiffness associated with more energy stored/dissipated in the adjoining area of the void, this can be understood as the facilitating mechanism for void growth.

\subsection{Experimental justifications}

To the surprise of Zhang et al. (1990), in their damage testing for measuring void growth in dual-phase steel sheets, they found that it was the plane strain loading case (where deformation was restricted along one of the two axial directions) that yielded the maximum void growth rate instead of being the biaxial stretching case.

Based on a simple form of the deformation type of plastic equations for continuum, that is

$$
\bar{\varepsilon}_{\mathrm{ij}}=\Psi \bar{S}_{\mathrm{ij}}
$$

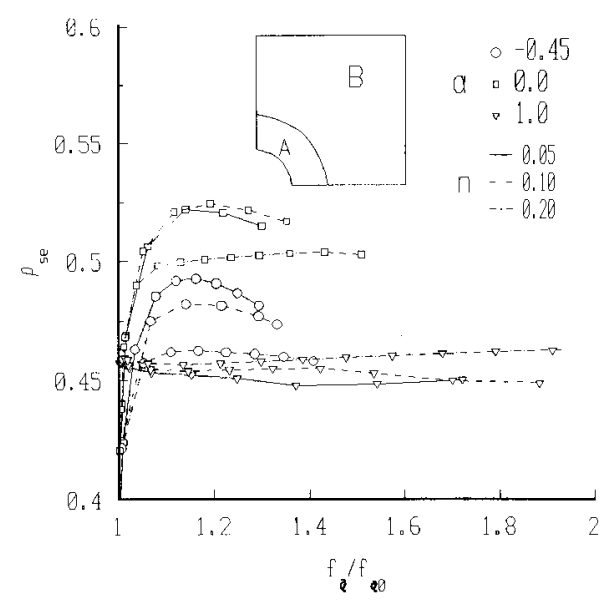

Fig. 9. The variation of the strain energy ratio $\rho_{\mathrm{se}}$ with respect to the void growth parameter $f_{\mathrm{a}} / f_{\mathrm{a} 0}$ (the exterior radius of the region $\mathrm{A}$ is $2 R_{0}$ ). 
where $\Psi=3_{\bar{\varepsilon}_{\mathrm{e}}} / 2_{\bar{\sigma}_{\mathrm{e}}}$ with the equivalent stress $\bar{\sigma}_{\mathrm{e}}=\sqrt{\frac{3}{2} \bar{S}_{\mathrm{ij}} \bar{S}_{\mathrm{ji}}}$ and the equivalent strain $\bar{\varepsilon}_{\mathrm{e}}=\sqrt{\frac{2}{3} \bar{\varepsilon}_{\mathrm{ij}} \bar{\varepsilon}_{\mathrm{ji}}}$ (when both elastic and volumetric strains are neglected. Zhang et al. (1990) calculated the triaxiality parameter for plane sheet $\left(\sigma_{3}=0\right)$ by the formula

$$
T=\bar{\sigma}_{\mathrm{m}} / \bar{\sigma}_{\mathrm{e}}=\frac{1+\alpha}{\sqrt{3}\left(1+\alpha+\alpha^{2}\right)^{1 / 2}}
$$

where $\alpha=\varepsilon_{2} / \varepsilon_{1}$. They had $\bar{\sigma}_{\mathrm{m}} / \bar{\sigma}_{\mathrm{e}}=0.666$ for the biaxial stretching $(\alpha=0.87)$, but 0.592 for the plane strain loading $(\alpha=0.055)$ and 0.366 for the uniaxial tension $(\alpha=-0.45)$. They took six or seven straining levels for each loading case until inhomogeneous instability deformation occurred. After reaching each straining level, the specimen was unloaded for measurements. They measured the average sizes of voids along both the rolling direction and the thickness direction of the specimens with respect to the equivalent strain. These measurements were necessary to exclude the influence of void nucleation and mainly to count the size growth of void. It was found that the plane strain loading yielded larger void size in both directions than those of the biaxial stretching. These facts proved that the plane strain loading case indeed had the fastest void growth rate, although its triaxiality was less than that of the biaxial stretching.

The maximum values of the triaxiality results given in Fig. 7(b) are in good agreement with those calculated by Eq. (4), which should be $T=0.667$ (biaxial, $\alpha=1$ ), 0.557 (plane strain, $\alpha=0$ ), and 0.336 (uniaxial tension, $\alpha=-0.45$ ). The fastest void growth given in Fig. 7(a) can provide an explanation for the question raised by the experimental observations of Zhang et al. (1990). Besides that, plane strain case is the favorable condition for localization to occur, which would incur void growth instability. This phenomenon had been reported by Li and Zhu (1995) and will be further addressed elsewhere with regard to the interaction between voiding nucleation and growth and material bifurcation.

\section{Conclusions}

The main conclusions of this paper can be given as follows:

(a) Large triaxiality can always stimulate large void growth, even if the overall equivalent strain are very small (e.g. the cases of $-0.3 \leqslant \alpha \leqslant 0$ in the axisymmetric cell). On the other hand, small or none void growth can be issued if triaxiality turns small or nil, although the overall equivalent strain can be large (e.g. the cases of $\alpha \leqslant-0.48$ in the axisymmetric cell).

(b) It is initially suggested to notice that, under the conditions of constant triaxiality or low triaxiality state, the straining mode characterized as the vanishing state of the third invariant of generalized strain rate most favors the growth of voids. This straining mode causes void growth instability in axisymmetric samples and faster void growth with lower triaxiality in plane stress samples under plane strain loading. 
(c) The straining mode comes into effect through influencing the strain concentration and the proportion of strain energy distributed in the adjoining layer encircling the void.

We do not intend to say much on the overall equivalent strain. It seems to us that it is likely to play such a role as a magnifying parameter, since it usually increases with the increase of triaxiality in the samples we studied here. Its influence on the nucleation of new voids is definite; however, this is not the purpose of our study in this paper.

\section{Acknowledgements}

The joint support of this work by the National Natural Science Foundation of China (Grant No. 19872064), the Chinese Academy of Science (KJ951-1-201) and that offered by the Laboratory for Nonlinear Mechanics of Continuous Media of the Institute of Mechanics are gratefully acknowledged.

\section{Appendix}

Comparisons between the plane stress cell model $\left(f_{\mathrm{a}}=6.5 \%\right.$ initially) and the three dimensional void model ( $f_{v}=6.5 \%$ initially) of Hom and McMeeking (1989) is shown in Fig. A1. The matrix material is taken to follow the same power law used by those authers as

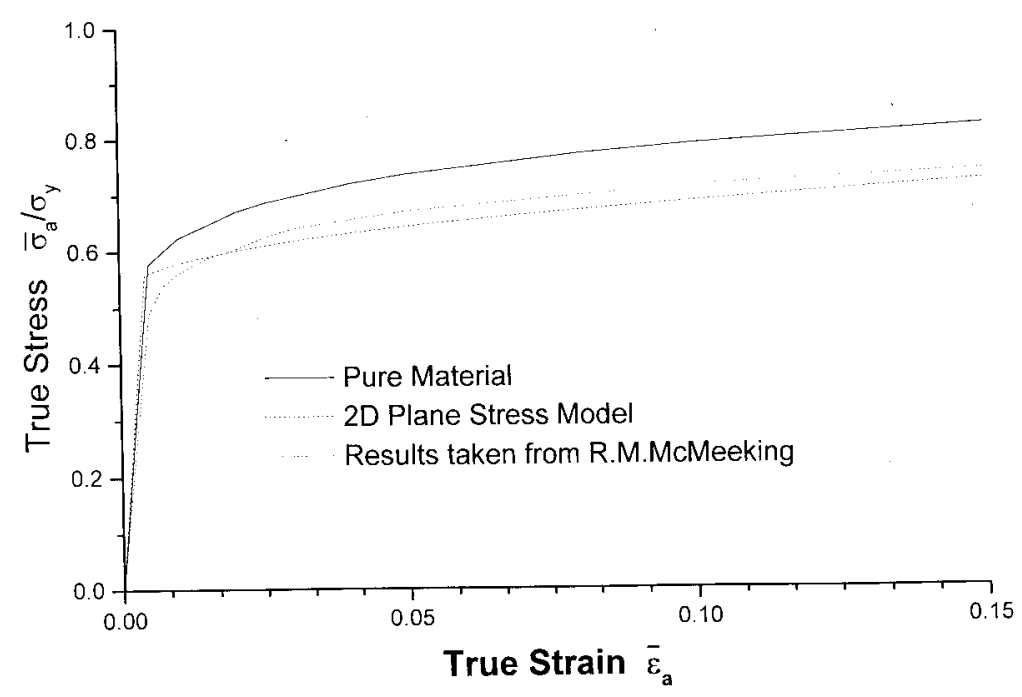

Fig. A1. Comparison of the true axial stress-strain curve $\bar{\sigma}_{\mathrm{a}}-\bar{\varepsilon}_{\mathrm{a}}$ between plane stress cell model and three dimensional cell model of Hom and McMeeking (1989). 


$$
\left(\frac{\sigma_{\mathrm{e}}}{\sigma_{\mathrm{y}}}\right)^{1 / N}-\frac{\sigma_{\mathrm{e}}}{\sigma_{\mathrm{y}}}=\frac{3 G \varepsilon_{\mathrm{e}}^{(p)}}{\sigma_{\mathrm{y}}}
$$

where $\sigma_{\mathrm{y}}$ is the yield stress, $G$ is the elastic shear modulus and $N$ is taken as 0.1 . In Fig. A1, $\bar{\sigma}_{\mathrm{a}}$ and $\bar{\varepsilon}_{\mathrm{a}}$ are the true tensile stress and strain loaded along the axial direction. $\bar{\sigma}_{\mathrm{a}}$ has equal absolute value as the compression stress loaded along the direction perpendicular to the axial axis in the pure shear condition. The stress-strain response is very near to the three dimensional modeling prediction given by Hom and McMeeking (1989).

\section{References}

Becker, R., 1987. The effect of porosity distribution on ductile fracture. J. Mech. Phys. Solids 35, 577-599.

Becker, R., Needleman, A., Richmond, O., Tvergaard, V., 1988. Void growth and failure in notched bars. J. Mech. Phys. Solids 36, 317-351.

Becker, R., Smelser, R.E., Richmond, O., Appleby, E.J., 1989. The effect of void shape on void growth and ductility in axisymmetric tension tests. Metall.Trans. A. 20A, 853-861.

Becker, R., Smelser, R.E., 1994. Simulation of strain localization and fracture between holes in an aluminum sheet. J. Mech. Phys. Solids 42, 773-793.

Benson, D.J., 1995. The effect of void cluster size on ductile fracture. Int. J. Plasticity 11, 571-582.

Briottet, L., Klocker, H., Montheillet, F., 1996. Damage in a viscoplastic material part 1: cavity growth. Int. J. Plasticity 12, 481-505.

Brocks, W., Sun, D.Z., Honig, A., 1995. Verification of the transferability of micromechanical parameters by cell model calculations with visco-plastic materials. Int. J. Plasticity 11, 971-989.

Fleck, N.A., Hutchinson, J.W., 1997. Strain gradient plasticity. In: Hutchinson, L.W., Wu, T.Y. (Eds.), Advances in Applied Mechanics, Vol. 33. Academia Press, New York, pp. 295-361.

Gurson, A.L., 1977. Continuum theory of ductile rupture by void nucleation and growth: part 1 - yield criteria and flow rules for porous ductile media. J. Engng Mater. Tech. 99, 2-15.

Hom, C.L., McMeeking, R.M., 1989. Void growth in elastic-plastic materials. J.A.M. 56, 309-317.

Huang, Y., Hutchinson, J.W., Tvergaard, V., 1991. Cavitation instabilities in elastic-plastic solids. J. Mech. Phys. Solids 39, 223-241.

Koplik, J., Needleman, A., 1988. Void growth and coalescence in porous plastic solids. Int. J. Solids Structures 24, 835-853.

Li, Guo-Chen, 1997. The challenges in the estimate of voiding damage. In: Khan, A.S. (Ed.), The 6th International Symposium on Plasticity and its Current Applications, Alaska, USA, 1997, Physics and Mechanics of Finite Plastic and Viscoplastic Deformation. Neat Press, Fultan, MD, pp. 205-206

Li, Guo-Chen, 1985a. Ductile behaviour of an ellipsoidal void model. Acta Mechanica Solida Sinica 3, 388-394 (in Chinese).

Li, Guo-Chen, 1985b. Dilatational plastic constitutive equations and their application to ductile fracture analysis of axisymmetric specimens. Acta Mechanica Sinica (English edition) 1, 49-58.

Li, G.C., Howard, I.C., 1983. The effect of strain softening in the matrix material during void growth. J. Mech. Phys. Solids 31, 85-102.

Li, G.C., Guennouni, T., Francois, D., 1989. Influence of secondary void damage in the matrix material around voids. Fatigue Fract. Engng Mater. Struct. 12, 105-122.

Li, G.C., Liu, H.Q., Du, M.L., Hong, Y.S., Zhang, X., 1992. Crack tip behaviour and crack propagation in ductile materials. Fatigue Fract. Engng Struct. 15, 187.

Li, Guo-Chen, Zhu, Chen, 1995. Formation of shear bands in plane sheet. Int. J. Plasticity 11, 605-622.

Magnusen, P.E., Dubensky, E.M., Koss, D.A., 1988. The effect of void arrays on void linking during ductile fracture. Acta Metall. 36, 1503-1509. 
McMeeking, R.M., Rice, J.R., 1975. Finite element formations for problems of large elastic-plastic deformation. Int. J. Solids Structures 11, 601-616.

Needleman, A., Tvergaard, V., 1984. An analysis of ductile rupture in notched bars. J. Mech. Phys. Solids $32,461-490$.

Ohno, N., Hutchinson, J.W., 1984. Plastic flow localization due to non-uniform void distribution. J. Mech. Phys. Solids 32, 63-85.

Rice, J.R., Tracey, D.M., 1969. On ductile enlargement of voids in triaxial stress fields. J. Mech. Phys. Solids 17, 201-217.

Tvergaard, V., 1981. Influence of voids on shear band instability under plane strain conditions. Int. J. Fracture 17, 389-407.

Worswick, M.J., Pick, R.J., 1990. Void growth and constitutive softening in a periodically voided solid. J. Mech. Phys. Solids 38, 601-626.

Yee, K.C., Mear, M.E., 1996. Effect of void shape on the macroscopic response of non-linear porous solids. Int. J. Plasticity 12, 45-68.

Zhang, Yi-Zeng, Hu, Xia-Yu, Huang, Ju-Qing, 1990. Damage in sheet forming of a dual-phase steel. Res. Mechanica 31, 35-48. 